\title{
The identity of the Spinola Collection Corixidae (Heteroptera)
}

\author{
Antti Jansson
}

\begin{abstract}
Jansson, A. 1992: The identity of the Spinola Collection Corixidae (Heteroptera). - Entomol. Fennica 3:173-175.

In a catalogue prepared on the Hemiptera of the Massimiliano Spinola Collection in the Torino Museum, Italy, 16 species were placed under the generic name Corixa, and two under the generic name Sigara. In ten of the taxa, the author was claimed to have been Spinola himself. However, only two valid taxa have been generally accepted, and the rest are either synonymic or only collection names. The present paper reveals the true identity of all the species of the Corixidae in the Spinola Collection.
\end{abstract}

Antti Jansson, Zoological Museum, University of Helsinki, P. Rautatiekatu 13, SF-00100 Helsinki, Finland

Casale (1981) published a catalogue on the Hemiptera of the Massimiliano Spinola Collection preserved in Torino Museum, Italy. This catalogue included altogether 18 taxa of Corixidae, 16 of which were placed under the generic name Corixa, and two under Sigara. Since the list included several previously unpublished names, I decided to investigate the material to find out the identity of the taxa. In the following, the taxa are listed in the order given by Casale (1981): each entry is as it appears in the catalogue with the code numbers followed by explanations of the codes (= the author of the taxon, collecting locality, origin of the material, and the number of specimens, i.e. data from the collection boxes, not necessarily affixed to pins of the specimens). Separated by a colon, the true identity of each taxon follows with possible data affixed to the pins. Notes on synonymy, taxonomic status and other possible information are given at the end of the entries.

\section{Genus Corixa}

fossarum, 44, 147, 20, 1 = Zetterstedt [correct author is Leach], Lapponia, Dahlbom, 1 ex.:
$10^{7}$ of Sigara striata (Linnaeus, 1758) with no label data affixed.

undulata, 391, 202, 20, 2 = Sahlberg [correct author is Fallén], Sweden, Dahlbom, 2 exx.: 1 q Sigara nigrolineata nigrolineata (Fieber, 1848) and $10^{7}$ S. semistriata (Fieber, 1848) with no label data affixed. - Note: Sigara undulata Fallén, 1814 is a synonym of S. striata (Linnaeus, 1758) (Jansson 1986b:72).

albiceps, 1, 53, 1, 1 = Spinola, Cap Bonne Espérance (= Cape of Good Hope), Buquet, 1 ex.: $10^{7}$ of Sigara wahlbergi Lundblad, 1928, with head, pronotum and fore legs missing, no label data affixed. - Note: C. albiceps is a collection name, description never published.

griseola, 1, 53, 1, 4 = Spinola, Cap Bonne Espérance (= Cape of Good Hope), Buquet, 4 exx.: $3 \sigma^{7} \sigma^{7}$ (one without abdomen and one with genital capsule glued separately on card) and 10 of Micronecta scutellaris pubescens Hutchinson, 1940, with no label data affixed. - Note: $C$. griseola is a collection name, description never published. Micronecta griseola Horváth, 1899, is a completely different and valid species.

carinata, 391, 202, 20, 1 = Sahlberg, Sweden, Dahlbom, 1 ex.: $10^{7}$ of Arctocorisa germari (Fieber, 1848) with no label data affixed; speci- 
men mostly eaten up by dermestids, but abdomen with genitalia remaining allow positive identification.

foveiceps [sic!], 1, 52, 32, 1 = Spinola (1852:234), Chile, Gay, 1 ex.: Sigara forciceps (Spinola), holotype male (described from a single specimen) with no label data affixed. - Note: Redescribed as Sigara rehi Jaczewski, 1930:147 and as Sigara argentiniensis chichinal Bachmann, 1960:38 (synonyms by Bachmann 1986:33).

hieroglyphica, 52, 208, 24, 5 and 52, 23, 95, 1 = Dufour, S. Séver, Dufour, 5 exx. and Dufour, Egypt, Waltl, 1 ex.: $10^{\top} 4 \propto \emptyset$ of Corixa punctata (Illiger, 1807), one of the females carrying handwritten labels "134 ?" and "Geoffroyi Fall. Suecia D. Dlbm” (= geoffroyi sensu Fallén from Sweden donated by Dahlbom) and $10^{7}$ of Corixa affinis Leach, 1817 with no label data affixed. Note: The label on the female from Sweden reveals that Casale (1981) had incorrect information about the collecting locality of this specimen. Corixa geoffroyi Leach, 1817 is a rejected and synonymic name for C. punctata (Illiger, 1807) (Opinion 281), while Corisa hieroglyphica Dufour, 1833 is a synonym of Sigara (Vermicorixa) lateralis (Leach, 1817) (China 1938).

striata, $1,5 / 125,0,4=$ Spinola $(1837: 57$ [1840:57]), Germany/Italy, origin unknown, 4 exx.: 10'1o of Hesperocorixa sahlbergi (Fieber, 1848) on one pin labeled "Suecia"; $10^{7}$ and 10 of $H$. sahlbergi on separate pins labeled "Allemagne" and "Italie"; $10^{7}$ of $H$. linnaei without label data. - Note: Altogether five specimens, which is one more than Casale (1981) listed, and also the collecting data (partly Sweden) differ from that given by Casale. Junior secondary homonym of Notonecta striata Linnaeus, 1758, no further action needed (Jansson 1986b).

schellembergii [sic!], 1, 125, 0, 2 = Spinola (1837:57 [1840:57]), Italy, origin unknown, 2 exx.: 1o Arctocorisa sp. (damaged) and 10 Callicorixa praeusta (Fieber, 1848) without label data. - Note: Spinola gave a replacement name to "Sigara striata Fabr." sensu Schellenberg (1800), which was conspecific with S. distincta (Fieber, 1848) (Jansson 1986b); the name was rejected as an unused senior synonym (Jansson 1987, Opinion 1524/1989).

immaculata, 1, 233, 35, 1: Spinola, Madagascar, Goudot, 1 ex.: 1@ Agraptocorixa gestroi
(Kirkaldy, 1898), right hemelytron missing and no label data affixed. - Note: C. immaculata is a collection name, description never published.

5-fasciata, 1, 44, 23, 1: Spinola, Bombay, Dupont, 1 ex.: 1o Sigara indica (Hutchinson, 1940) with no label data affixed. - Note: $C$. quinquefasciata is a collection name, description never published.

dorsimaculata, 1, 154, 23, 1 = Spinola, Mexico, Dupont, 1 ex.: $1 \propto$ Corisella mercenaria (Say, 1832) with no label data. - Note: $C$. dorsimaculata is a collection name, description never published.

minuta, 59, 125/33, 40, 5 = Fabricius, Bavaria/ Italy, Gribodo, 5 exx.: $10^{7}$ Sigara limitata limitata (Fieber, 1848) with hand-written label "Corixa regularis H.Sch. Germania", and $30^{7} \mathrm{O}^{7} 1$ 1o Sigara lateralis (Leach, 1817) with no label data. Note: Sigara minuta Fabricius, 1794, is conspecific with Micronecta griseola Horváth, 1899, but has been rejected as an unused senior synonym (Jansson 1986a, Opinion 1491/1988). Corixa regularis Herrich-Schäffer, 1853, is a junior synonym of Hesperocorixa linnaei (Fieber, 1848) (Westhoff 1880, Jaczewski 1939).

hellensis [sic!], 391, 33, 78, 2 = Sahlberg, Bavaria, Sturm, 2 exx.: 1\% of Sigara hellensii (C. Sahlberg, 1819) and $10^{7}$ of S. semistriata (Fieber, 1848 ) with no label data on either specimen.

coleoptrata, 59, 33, 78/40, 4 = Fabricius, Bavaria, Sturm/Gribodo, 4 exx.: $10^{7}$ and 300 . Cymatia coleoptrata (Fabricius, 1777), one of the females with the label "Suecia", others without label data. - Note: Collecting locality (at least one specimen from Sweden) differs from that given by Casale (1981).

antillarum, 1, 27, 22, 3 = Spinola, Antilles, Deyrolles, 3 exx.: $20^{7} \sigma^{7}$ and 10 Centrocorisa nigripennis (Fabricius, 1803) without label data. - Note: C. antillarum is a collection name, description never published.

\section{Genus Sigara}

minutissima, 144, 207, 0, 2 = Linnaeus, Sardinia, origin unknown, 2 exx.: $10^{\top 1} 1$ of Micronecta scholtzi (Fieber, 1860) without label data.

leucocephala, 1, 207, 36, 2: Spinola (1837:59 [1840:59]), Sardinia, Gené, 2 exx.: Micronecta 
leucocephala (Spinola, 1837) lectotype and paralectotype (Jansson 1986b:14), both brachypterous females with type status designation labels but no further data.

Acknowledgements. I wish to thank Dr. P. M. Giachino for mailing the Spinola Collection specimens to me for investigation.

\section{References}

Bachmann, A. O. 1960 (1959 issue): Notas sobre Corixidae. - Rev. Soc. Entomol. Argentina 22:34-40.

- 1986 (1985 issue): Notas sobre Corixidae (Heteroptera) (Quinta serie). — Rev. Soc. Entomol. Argentina $44: 33-36$

Casale, A. 1981: Cataloghi II — Collezione Emitterologica di Massimiliano Spinola. - Museo Regionale di Scienze Naturali, Torino, $120 \mathrm{pp}$.

China, W. E. 1938: Notes on the nomenclature of British Corixidae. — Entomol. Mon. Mag. 74:34-39.

Dufour, L. 1833: Recherches anatomiques et physiologiques sur les Hémiptères. - Mem. Sav. Acad. R. Sci. Inst. France 4:129-461.

Fabricius, J. C. 1777: Genera insectorum eorumque characteres naturales secundum numerum, figuram, situm et proportionom omnium partium oris adiecta mantissa specieram nuper detectarum. - M. F. Bartschii, Chilonii, 310 pp.

- 1794: Entomologia systematica, emendata et aucta, secundum classes, ordines, genera, species. Tom. 4. - Hafniae, 472 pp.

- 1803: Systema Rhyngotorum. - Brunsvigae, 105 pp.

Fallén, C. F. 1814: Hydrocorides et Naucorides Sveciae. - Berlingianis, Lundae, 8 pp.

Fieber, F. X. 1848: Synopsis aller bisher in Europa entdecten Arten der Gattung Corisa. - Bull. Soc. Int. Nat. Moscou 21:505-593.

- 1860 (1861): Die europäischen Hemiptera. - Verl. Carl Gerold's Sohn, Wien, 444 pp.

Herrich-Schäffer, G. A. W. 1853: Die wanzenartigen Insekten. Bd. 9. - Nürnberg, 348 pp.

Horváth, A. 1899: Synopsis des Micronecta paléarctiques. — Rev. d'Entomol. 18:101-104.

Hutchinson, G. E. 1940: Revision of the Corixidae of India and adjacent regions. - Trans. Connecticut Acad. Arts Sci. 33:339-476.

Illiger, C. 1807: Fauna etrusca sistens insecta quae in provinciis Florentina et Pisana presentim collegit Petrus Rossius. Iterum edita et perpetuis aucta. Tom. 2. Fleckeisen, Helmstadt, 511 pp.

Jaczewski, T. 1930: Die Corixiden (Corixidae, Heteroptera) des Zoologischen Staatsinstituts und Zoologischen Museums in Hamburg. - Mitt. Zool. Staatsinst. Zool. Mus. Hamburg 44:140-148.
- 1939: Notes on Corixidae. XV-XXIII. - Ann. Mus. Zool. Polon. 13:269-302.

Jansson, A. 1986a: Micronecta griseola Horváth, 1899 (Insecta, Heteroptera, Corixidae): Proposed conservation by the suppression of Sigara minuta Fabricius, 1794 and Sigara lemana Fieber, 1860. — Bull. Zool. Nom. 45:165-166.

- 1986b: The Corixidae (Heteroptera) of Europe and some adjacent regions. - Acta Entomol. Fennica 47:1-94.

- 1987: Corisa distincta Fieber, 1848 (currently Sigara (Subsigara) distincta; Insecta, Hemiptera): proposed conservation of the specific name. - Bull. Zool. Nom. 44:101-102.

Kirkaldy, G. W. 1898: Note sopra alcuni rincoti acquatici africani dell Museo Civico di Storia Naturale di Genova. - Ann. Mus. Civ. Stor. Nat. Giacomo Doria 39:144-145.

Leach, W. E. 1817: On the classification of the natural tribe of insects Notonectides, with descriptions of British species. - Trans. Linn. Soc. London 12:10-18.

Linnaeus, C. 1758: Systema Nature, 10th ed. - Holmiae, 823 pp.

Lundblad, O. 1928: Beitrag zur Kenntnis der Corixiden. — Entomol. Tidskr. 48:219-243.

Opinion 281/1954: Validation, under the plenary powers, of the generic name "Corixa" Geoffrey, 1762 (Class Insecta, Order Hemiptera), - Op. Decl. Int. Comm. Zool. Nom. 6:205-223.

Opinion 1491/1988: Micronecta griseola Horvaéth, 1899 (Insecta, Heteroptera): specific name conserved. Bull. Zool. Nom. 45:165-166.

Opinion 1524/1989: Corisa distincta Fieber, 1848 (currently Sigara (Subsigara) distincta; Insecta, Hemiptera): specific name conserved. - Bull. Zool. Nom. 46:64.

Sahlberg, C. R. 1319: Observationes quasdam historiam Notonectidum, imprimis Fennicarum, illustrantes. Diss. Acad., Typis Frenckelliorum, Aboae, 15 pp.

Say, T. 1832: Descriptions of new species of Heteropterous Hemiptera of North America. - New Harmony, Indiana, $39 \mathrm{pp}$.

Schellenberg, J. R. 1800: Das Geschlecht der Land und Wasserwanzen. - Orell, Füssli \& Co., Zürich, 32 pp.

Spinola, M. 1837 [1840]: Essai sur les genres d'insectes appartenants á l'ordre des Hémiptères, Lin. ou Rhyngotes, Fab. et á la section des Hétéroptères, Dufour [second printing with slightly different title in 1840: Essai sur les insectes Hémiptères, Rhyngotes, ou Héteéroptères]. - Y. Gravier, Génes, 383 pp. [J.-B. Baillière, Paris, 383 pp.].

- 1852: Hemiptera. - Gay, C.: Historia fisica y politica de Chile. Insecta 7:113-320.

Westhoff, F. 1880: Verzeichnis bisher in Westfalen aufgefundener Arten aus der Gruppe: Hemiptera Heteroptera. - Westfäl. Prov.-Ver. Wiss. Kunst 8:55-64.

Received 23.XII.1991 